\title{
Multiple lesions in cerebral white matter in two young adults with thoracic extramedullary tumours
}

\author{
Fabrizio Salvi, Mario Mascalchi, Rosaria Plasmati, Roberto Michelucci, Fabio Calbucci, \\ Giancarlo Dal Pozzo, Carlo Alberto Tassinari
}

University of Bologna, Bellaria Hospital,

Bologna

Division of Neurology

F Salvi

R Plasmati

R Michelucci

C A Tassinari

Division of

Neurosurgery

F Calbucci

Neuroradiology,

Neurological Sciences,

University of

Florence, Florence

M Mascalchi

G Dal Pozzo

Correspondence to:

Dr M Mascalchi,

Department of

Neurology, University of

Florence, Viale Morgagni

85, 50134 Florence, Italy.

Received 8 April 1991

and in revised form

24 June 1991.
Department of

Accepted 4 July 1991

\begin{abstract}
Cranial MRI showed multiple lesions in white matter that were thought to be consistent with multiple sclerosis in two young adults presenting with symptoms of progressive myelopathy. MRI of the cervicothoracic spine around one and two years after onset showed the myelopathy to be due to mid-thoracic tumours. The tumours (an extradural meningioma and intradural neuroma) were resected with complete resolution of myelopathy in one patient but no recovery in the other. Spinal MRI (or myelography) should be performed in young patients presenting with signs of progressive myelopathy even when cranial MRI shows a picture typical of multiple sclerosis.
\end{abstract}

Multiple sclerosis ranks first among the nontraumatic causes of myelopathy in young adults. ${ }^{1}$ Although the relapsing remitting course of the disease usually distinguishes patients with multiple sclerosis related myelopathy from those with other aetiologies, the existence of a primary chronic progressive
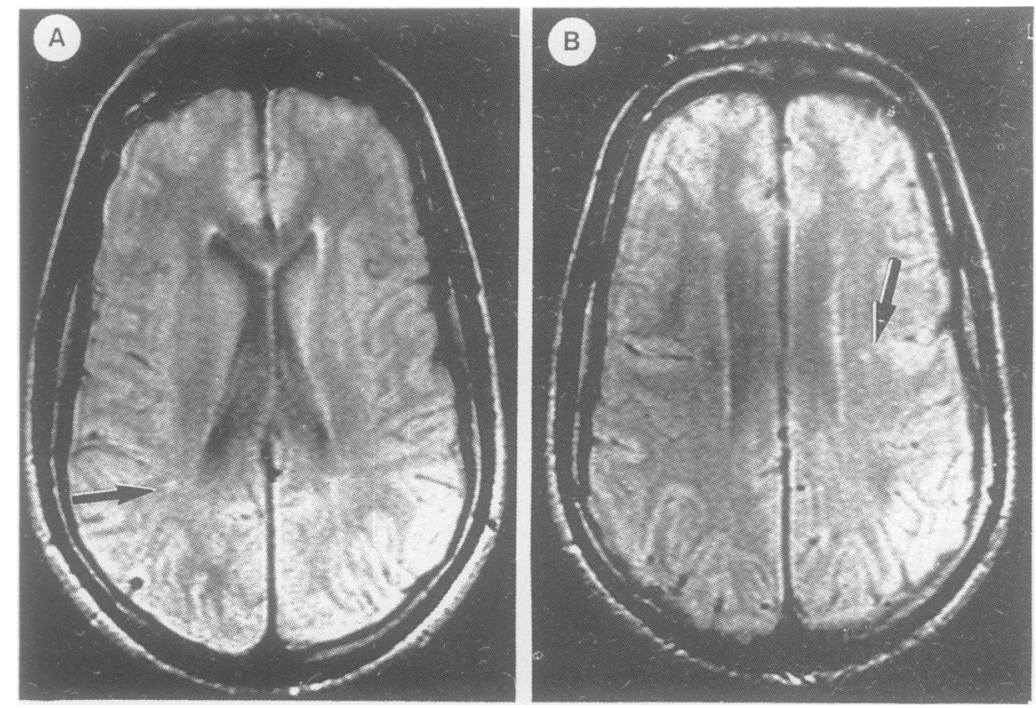

Figure $1(a)$, (b) Case 1. Multiple tiny foci of high signal intensity in the deep cerebral white matter (arrows) (SE TR $1510 \mathrm{~ms}$; TE $50 \mathrm{~ms}$ ). form of multiple sclerosis related myelopathy is well known. ${ }^{2}$

Spinal MRI has replaced myelography in investigating possible structural changes in patients with progressive myelopathies. In young adults such lesions are represented predominantly by intramedullary tumours, whereas extramedullary tumours are rather uncommon. $^{3}$

Cranial MRI has been considered sufficient to diagnose multiple sclerosis related myelopathy by detecting multiple lesions in white matter in patients with negative results in investigations for spinal disease. ${ }^{24-6}$

We report the cases of progressive myelopathy in two young adults in which a diagnosis of multiple sclerosis related myelopathy-supported by multiple focal lesions in white matter on cranial MRI-was subsequently shown to be incorrect on spinal MRI.

\section{Case reports}

Case 1

A 20 year old man presented in April 1989 with increasing difficulty in running and a sensation of tight legs. A neurological examination at another hospital showed a mild spastic paraparesis, nystagmus on extreme lateral gaze, and decreased vibrational sense in his right leg. He had cranial MRI ( $0.5 \mathrm{~T})$, which detected multiple tiny lesions in the subcortical and periventricular white matter (figure 1); these were thought to be consistent with multiple sclerosis. Visual evoked potentials were unremarkable. Despite steroid treatment the weakness in his lower limbs worsened, and in November 1989 he noted urinary urgency and an electrical feeling down the spine when he flexed his head. In January 1990 neurological examination showed severe paraparesis with Babinski's sign bilaterally, clonus of the right ankle, absent abdominal reflexes, and decreased pain sensation to the lower limbs. Somatosensory evoked potentials showed a slight delay in the latency of the response after stimulation of the left median nerve and complete absence of the evoked response after stimulation of his lower limbs. 


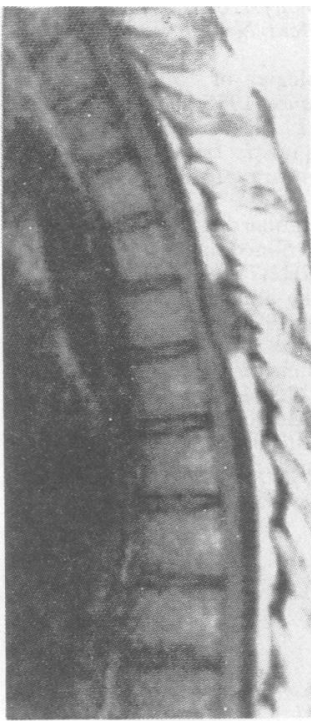

Figure 2 Case 1. MRI of the thoracic spine. On a $T 1$ weighted $S E$ image (TR $360 \mathrm{~ms}, T E 29 \mathrm{~ms}$ ) an extramedullary mass is detected within the spinal canal at the level of $T 5$ and impinges posteriorly on the cord. The mass appears isointense to the cord and markedly hypointense to the posterior epidural fat.

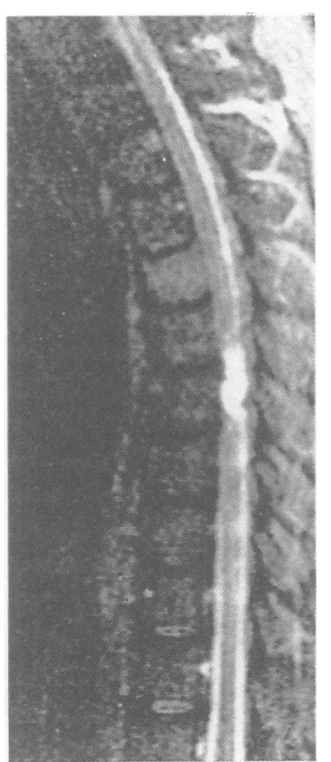

Figure 4 Case 2. A mass hyperintense to the norma cord is present within the spinal canal at level T7 on a $T 2$ weighted $S E$ image (TR 1520 ms, TE 200

$m s)$.

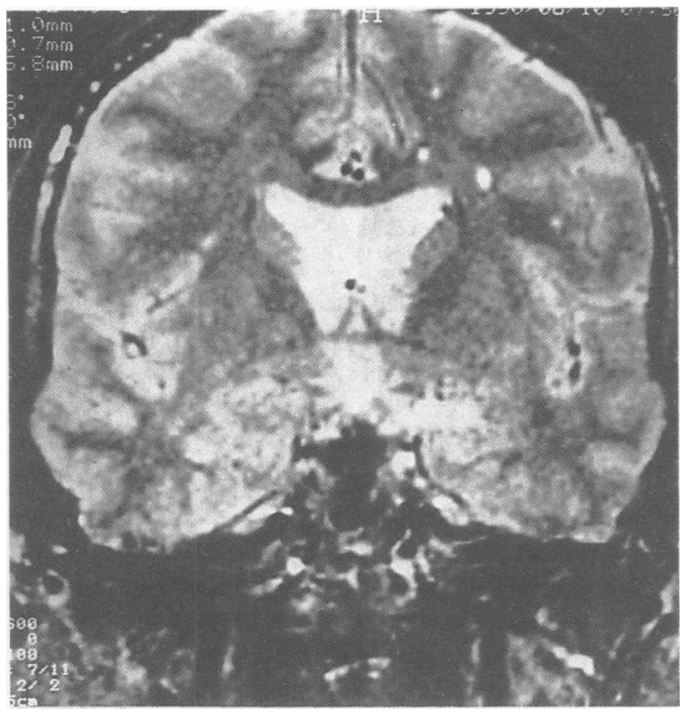

Figure 3 Case 2. Multiple small foci areas of high signal intensity are identifiable within the periventricular and subcortical white matter on a T2 weighted $S E$ image (TR $1600 \mathrm{~ms}$, TE $100 \mathrm{~ms}$ ).

In March 1990 he was almost unable to walk, even with help; he had MRI of the cervicothoracic spine $(0.5 \mathrm{~T})$, which showed an extradural mass at $\mathrm{T} 5$ that substituted the epidural fat and impinged posteriorly on the cord (figure 2). Myelography with water soluble contrast medium showed complete block of the iodinated column at the $\mathrm{T} 4-\mathrm{T} 5$ intervertebral space, suggesting an extradural mass. Surgery was performed eight days after spinal MRI, and an extradural mass involving the right T4 root was removed. Histological examination showed a transitional meningioma. The patient showed prompt improvement of the paraparesis postoperatively, with complete recovery of function in January 1991.

\section{Case 2}

A 32 year old man had had pain in his left abdomen for about two years; it progressively extended to his left hip and leg and was thereafter accompanied by weakness of his left lower limb. During the two months before admission in September 1990 weakness extended to his right lower limb and he noted urinary urgency. Cranial MRI in August 1990 with an $0.5 \mathrm{~T}$ machine showed multiple small lesions in the cerebral white matter (figure 3 ) that were thought to be consistent with multiple sclerosis. At admission the paraparesis confined him to a wheelchair. Neurological examination showed brisk reflexes in his lower limbs, with Babinski's sign bilaterally and a level of complete sensory deficit at T10. In addition, he presented with a non-paralytic, horizontal strabismus (present since his childhood, as proved in some of his old photographs), and a bilateral myopic choroid crescent was detected on fundoscopy. Thoracic MRI (0.5 T) showed an extramedullary lesion at T7 (figure 4). Although the intradural mass originating from the left T8 posterior route was completely excised at surgery nine days after spinal MRI, recovery of function was incomplete in his lower limbs at discharge in November 1990. Histological examination showed a neuroma.

\section{Discussion}

The occurrence of monosymptomatic progressive spastic myelopathy of unknown aetiology is a comparatively common diagnostic dilemma in clinical practice. Based on the results of a necropsy series, Marshall in 1955 pointed out that multiple sclerosis is one of the main causes of these symptoms. ${ }^{7}$ In contrast to structural myelopathies, however, primary progressive demyelinating myelopathy predominantly affects middle aged people and is characterised clinically by a progressive neurological deficit over several years, an early onset of disturbances of bladder function, and rarity of radicular pain or level of sensory deficit. ${ }^{12} \mathrm{~A}$ differential diagnosis based solely on clinical findings may be difficult in some cases. Strategies therefore have been developed to increase the detection of cases related to multiple sclerosis myelopathy by using paraclinical tests. These include several neurophysiological investigations and cranial MRI-with the aim of detecting additional, clinically silent lesions of white matter ${ }^{24-6}$ - and specialised laboratory techniques such as agarose electrophoresis to detect characteristic inflammatory abnormalities of the cerebrospinal fluid. ${ }^{8}$

Disappointingly, cerebrospinal fluid was not analysed in our two patients with progressive myelopathy; however, in both, cranial MRI showed multiple focal lesions in cerebral white matter, which made a diagnosis of multiple sclerosis related myelopathy probable. Although spinal MRI makes possible visualisation of spinal cord plaques in an appreciable number of patients presenting with demyelinating myelopathies, ${ }^{9}$ this possibility was initially discarded in our cases. This deferred the spinal MRI examination that detected extramedullary tumours as the cause of myelopathy and permitted the patient in case 1 , with the shorter interval between onset and surgery, a complete recovery of the compressive myelopathy after removal of the mass. Focal lesions in white matter detected by cranial MRI are a non-specific finding in that they have been reported in several healthy elderly volunteers ${ }^{10}$ and have several underlying pathological changes, including demyelination plaques, microinfarcts, gliosis, and dilated perivascular spaces. ${ }^{10}$ Their observation in young adults is, however, uncommon ${ }^{11}$ and strongly supports a diagnosis of multiple sclerosis. $^{12}$ Moreover, their detection in patients with unexplained myelopathy has been considered to be an important clue in diagnosing multiple sclerosis related myelopathy. ${ }^{24-6}$

Since we have no pathological correlation of the white matter lesions observed on cranial MRI in our cases we cannot establish their cause; even the hypothesis that they correspond to demyelination foci of multiple sclerosis, and hence that these patients had a dual pathology, cannot be ruled out.

However, from a practical point of view, their interpretation as an indirect clue to the inflammatory origin of the progressive myelopathy in our cases proved to be wrong and misleading. This supports the opinion that spinal MRI or, if this is not available, myelography should be performed early in 
young patients with progressive myelopathy irrespective of the results of cranial MRI.

1 Adams RD, Victor M. Principles of neurology. 3rd ed. New York; McGraw-Hill, 1985.

2 Weinshenker BG, Gilbert JJ, Ebers GC. Some clinical and pathologic observations on chronic myelopathy: a variant of multiple sclerosis. J Neurol Neurosurg Psychiatry

3 Enzmann DR, DeLaPaz RL. Tumor: Enzmann DR, DeLaPaz RL, Rubin J, eds. Magnetic resonance of the spine, Morsby, St Louis, Missouri: 1990;301-422.

4 Edwards MK, Farlow MR, Stevens JC. Cranial MR in spinal cord MS: diagnosing patients with isolated spinal cord syndromes. AJNR 1986;7:1003-5.

5 Miska RM, Pojunas KW, McQuillen MP. Cranial magnetic resonance imaging in the evaluation of myelopathy of undetermined origin. Neurology 1987;37:840-3.

6 Marti-Fabregas J, Martinez JM, Illa I, Escartin A.
Myelopathy of unknown etiology. A clinical follow-up and MRI study of 57 cases. Acta Neurol Scand 1989;80 455-60.

7 Marshall J. Spastic paraplegia of middle age. A clinicopathological study. Lancet 1955;i:643-6.

8 Poser CM, Paty D, Scheinberg L, McDonald WI, Davis FA, Ebers GC, et al. New diagnostic criteria for multiple sclerosis: guidelines for diagnostic protocols. Ann Neurol 1983;13:227-31.

9 Miller DH, McDonald WI, Blumhardt LD, et al. Magnetic resonance imaging in isolated noncompressive spinal cord syndromes. Ann Neurol 1987;22:714-23.

10 Mascalchi $M$, Inzitari D. Leuko-araiosis: a reappraisal. II MR studies. Ital J Neurol Sci 1991;12:271-9.

11 Fazekas F. Magnetic resonance signal abnormalities in asymptomatic individuals: their incidence and functional correlates. Eur Neurol 1989;29:164-8.

12 Ormerod IEC, Miller DH, McDonald WI, Du Boula EPGH, Rudge P, Kendall BE, et al. The role of NMR imaging in the assessment of multiple sclerosis and isolated neurological lesions. A quantitative study. Brain 1987;110:1579-616. 